\title{
Does Cyclic ADP-Ribose (cADPR) Activate the Non-selective Cation Channel TRPM2?
}

\author{
Ralf Fliegert, Winnie M. Riekehr and Andreas H. Guse* \\ The Calcium Signalling Group, Department of Biochemistry and Molecular Cell Biology, University Medical Center \\ Hamburg-Eppendorf, Hamburg, Germany
}

TRPM2 is a non-selective, $\mathrm{Ca}^{2+}$-permeable cation channel widely expressed in immune cells. It is firmly established that the channel can be activated by intracellular adenosine 5 '-diphosphoribose (ADPR). Until recent cryo-EM structures have exhibited an additional nucleotide binding site in the $\mathrm{N}$-terminus of the channel, this activation was thought to occur via binding to a C-terminal domain of the channel that is highly homologous to the ADPR pyrophosphatase NudT9. Over the years it has been controversially discussed whether the $\mathrm{Ca}^{2+}$ mobilizing second messenger cyclic ADP ribose (CADPR) might also directly activate $\mathrm{Ca}^{2+}$ entry via TRPM2. Here we will review the status of this discussion.

OPEN ACCESS

Edited by:

Santiago Partida Sanchez, Nationwide Children's Hospital,

United States

Reviewed by:

Yasuo Mori,

Kyoto University, Japan

Wei Yang,

Zhejiang University, China Tomohiro Numata,

Fukuoka University, Japan

*Correspondence:

Andreas H. Guse guse@uke.de

Specialty section:

This article was submitted to Molecular Innate Immunity, a section of the journal

Frontiers in Immunology

Received: 01 April 2020

Accepted: 24 July 2020

Published: 11 August 2020

Citation:

Fliegert R, Riekehr WM and Guse AH (2020) Does Cyclic ADP-Ribose (cADPR) Activate the Non-selective Cation Channel TRPM2? Front. Immunol. 11:2018. doi: 10.3389/fimmu.2020.02018
Keywords: TRPM2, ion channel, calcium, signal transduction, cADPR

\section{INTRODUCTION}

TRPM2 is a non-selective, $\mathrm{Ca}^{2+}$-permeable cation channel expressed in immune cells like monocytes $(1,2)$, macrophages (3-5), neutrophils (6-9), dendritic cells (10) and effector T cells (11). The channel plays a role in the inflammatory response by modulating differentiation (10), cell migration and chemotaxis $(7,10,12)$, cytokine (11) and chemokine secretion (1) and is regulated in a complex manner integrating inputs from the physical environment of the cell like temperature (13) and $\mathrm{pH}(14)$ with intracellular second messengers like $\mathrm{Ca}^{2+}(15,16)$ and adenine nucleotides. Since cloning of TRPM2 over 20 years ago (17), a number of adenine nucleotides have been proposed to affect TRPM2. While ADPR and 2'-deoxy-ADPR (18) are firmly established as TRPM2 agonists, the roles of NAADP and CADPR in activation of the channel remain controversial. In this review we want to summarize the literature regarding the role cADPR with an emphasis on recent (structural) data.

In 2001 Perraud et al. found that the cytosolic C-terminus of TRPM2 (at that time known as LTRPC2) contains a Nudix box motif (19). This sequence motif is known from a huge superfamily of proteins, many of them pyrophosphorylases that hydrolyse "nucleoside diphosphates linked to a residue X" (hence the name NudiX) [reviewed in Srouji et al. (20)]. Nudix pyrophosphorylases differ largely with regard to substrate specificity with some of them having only a single substrate while others hydrolyse a broad range of dinucleotides. By sequence analysis Perraud et al. discovered the gene for an enzyme, now known as NudT9, that exhibits $50 \%$ sequence homology to TRPM2. By testing a number of potential substrates, they identified adenosine $5^{\prime}$-diphosphoribose (ADPR) as its substrate (19). ADPR is a cellular nucleotide that can arise from hydrolysis of NAD by the NAD glycohydrolase CD38 $(21,22)$ or may be cleaved from poly-ADP-ribosylated or mono-ADP-ribosylated proteins (23). Perraud et al. also demonstrated that ADPR can activate TRPM2 in a $\mathrm{Ca}^{2+}$-dependent manner supposedly by binding to its C-terminal NudT9 homology domain (19). 
Kolisek et al. later found that cyclic ADP ribose (cADPR), another metabolite of NAD, is also able to activate TRPM2 (24). CADPR is a second messenger in a number of different cell types [reviewed in Lee (25)] including cells of the immune system like $\mathrm{T}$ cells (26) and neutrophils (27) that mobilizes $\mathrm{Ca}^{2+}$ from intracellular stores (26). The cellular target for cADPR remains still elusive. While photoaffinity labeling with a cADPR analog in sea urchins pointed to a receptor with a molecular weight of 100$140 \mathrm{kDa}$ which has so far escaped identification (28), most data for higher animals indicate that $\mathrm{Ca}^{2+}$ release by cADPR involves ryanodine receptors type 2 (29) or type 3 (30). This might be by displacement of FKBP12.6 from the ryanodine receptors resulting in an increased open probability $(31,32)$ or by indirect mechanisms like increasing store loading by stimulating activity of the ER $\mathrm{Ca}^{2+}$ pump SERCA (33-35).

In addition to releasing $\mathrm{Ca}^{2+}$ from intracellular stores, it has also been shown that CADPR can trigger $\mathrm{Ca}^{2+}$ entry via the plasma membrane. In Jurkat $\mathrm{T}$ cells microinjection of cADPR activates $\mathrm{Ca}^{2+}$ entry over the plasma membrane (36) and in neutrophiles the cADPR antagonist 8-Br-cADPR inhibits $\mathrm{Ca}^{2+}$ influx in response to the chemotactic peptide fMLP (27). So far it is unclear whether this $\mathrm{Ca}^{2+}$ influx works via activation of capacitative $\mathrm{Ca}^{2+}$ entry via the STIM/Orai system (37) or involves additional $\mathrm{Ca}^{2+}$ channels directly activated by cADPR. These findings made the observation that cADPR might activate TRPM2 especially interesting.

Activation of TRPM2 by cADPR requires exceedingly high concentrations $\left(\mathrm{EC}_{50} 700 \mu \mathrm{M}\right)$ of $\mathrm{cADPR}$ and even at $3 \mathrm{mM}$ CADPR in the patch pipette the current was only about $5 \%$ of the current evoked by ADPR in low micromolar concentrations (24). Cellular concentrations of cADPR determined in the past by us and others using either $\operatorname{HPLC}(26,38)$ or an enzymatic cycling assay (39-41) are significantly lower. This makes it highly unlikely that CADPR alone can contribute to $\mathrm{Ca}^{2+}$ entry by activation of TRPM2. On the other hand did CADPR shift the concentration-response for ADPR by two orders of magnitude from an $\mathrm{EC}_{50}$ of $12 \mu \mathrm{M}$ in the absence of cADPR to $90 \mathrm{nM}$ in the presence of $10 \mu \mathrm{M}$ cADPR, resulting in the hypothesis that cADPR and ADPR may activate TRPM2 synergistically (24).

\section{POTENTIAL BINDING SITE OF CADPR AT TRPM2}

A synergism between $\mathrm{CADPR}$ and $\mathrm{ADPR}$ raises the question of the binding site. 8-Br-cADPR, an antagonist of cADPR (42) inhibited activation of TRPM2 by CADPR but not by ADPR, whereas AMP, one of the products of the enzyme NudT9, inhibited activation by ADPR but not by CADPR, indicating that ADPR and CADPR do not act via the same binding site. Since AMP affects activation by ADPR it seems that ADPR binds to the NudT9H domain, whereas cADPR would bind to a distinct site for which it competes with 8-Br-cADPR. First indications of a secondary nucleotide binding site came from work on TRPM2 from the sea anemone Nematostella vectensis (nvTRPM2). nvTRPM2 also features a Nudix domain, but Kühn et al. showed that removal of this NudT9H domain does not interfere with gating of the channel by ADPR, but that the domain is catalytically active and breaks down ADPR (43). This led them to propose that the ADPR binding site for nvTRPM2 is separate from the NudT9H domain. Later they showed that the NudT9H domain of nvTRPM2 while not required for activation by $\mathrm{ADPR}$ can contribute to gating. While in nVTRPM2, activation by ADPR is not affected by removal of the NudT9H domain, activation by inosine $5^{\prime}$-diphosphoribose (IDPR) is abrogated. This shows that the second binding sites can modulate channel activity and exhibits a different agonist selectivity (44). During the last year a number of cryo-EM structures of TRPM2 from different species became available (4548). One especially interesting finding from the studies by Huang et al. was the identification of additional nucleotide binding sites in TRPM2 from zebra fish (drTRPM2) (47) and humans (48). Located between the first two melastatin homology regions (MHR1/MHR2) in the cytosolic N-terminus of the channel they observed an ADPR molecule in a horseshoe-like conformation that resembles the conformation of CADPR in both zebra fish and human TRPM2 (Figure 1). In human TRPM2 they were able to resolve an additional ADPR molecule in the NudT9H domain which exhibited in contrast to the horse-shoe-like ADPR in the MHR1/MHR2 binding site an elongated confirmation (Figure 1). They also solved a structure of TRPM2 in an inhibited state with the cADPR antagonist 8-Br-cADPR in the presence of $\mathrm{Ca}^{2+}$. The functional role of these distinct nucleotide binding sites remains controversial. While Huang et al. observed a loss of activity when mutating the MHR1/MHR2 binding site as well as when removing the NudT9H domain in both zebra fish (47) as well as human TRPM2 (48), Wang et al. did neither observe any ADPR related electron density in the MHR1/MHR2 domain nor did they see an effect of the mutation of this site (46).

\section{TEMPERATURE DEPENDENCY OF CADPR MEDIATED TRPM2 ACTIVATION}

Togashi et al. first noticed that TRPM2 can be activated by heat above a threshold temperature of $35^{\circ} \mathrm{C}$ with currents increasing up to $42^{\circ} \mathrm{C}$ (13). While ADPR activated TRPM2 already at $25^{\circ} \mathrm{C}$, the currents were largely enhanced when the temperature was increased to $35^{\circ} \mathrm{C}$ and above. In contrast to Kolisek et al. Togashi et al. did not observe any cADPR evoked currents at $25^{\circ} \mathrm{C}$ but found that $100 \mu \mathrm{M}$ cADPR in the pipette largely enhanced TRPM2 currents evoked by heat. This effect was absent in cells expressing a TRPM2 variant lacking the NudT9H domain (13).

Due to its labile N1-glycosidic bond cADPR is prone to hydrolysis to ADPR. At room temperature and under slightly acidic conditions its half-life is 10 days which decreases to $24 \mathrm{~h}$ at $37^{\circ} \mathrm{C}(49)$. Even frozen solutions of cADPR have been shown to slowly degrade to ADPR at $-20^{\circ} \mathrm{C}(50)$. cADPR is also subject to enzymatic hydrolysis to ADPR by $\mathrm{CD} 38$ and CD157/Bst-1 which besides NAD glycohydrolase and ADPribosyl cyclase activity also exhibit cADPR hydrolase activity (5153). Hydrolysis of the pyrophosphate in cADPR by an $\mathrm{Mn}^{2+}$ dependent ADP-ribose/CDP-alcohol pyrophosphatase yields $\mathrm{N}^{1}$ (5-phosphoribosyl)-AMP resulting in breakdown of cADPR 


\section{N MHR1/2 MHR3 MHR4 preS1 TM-Region TRP Rib Pole NUDT9H C}

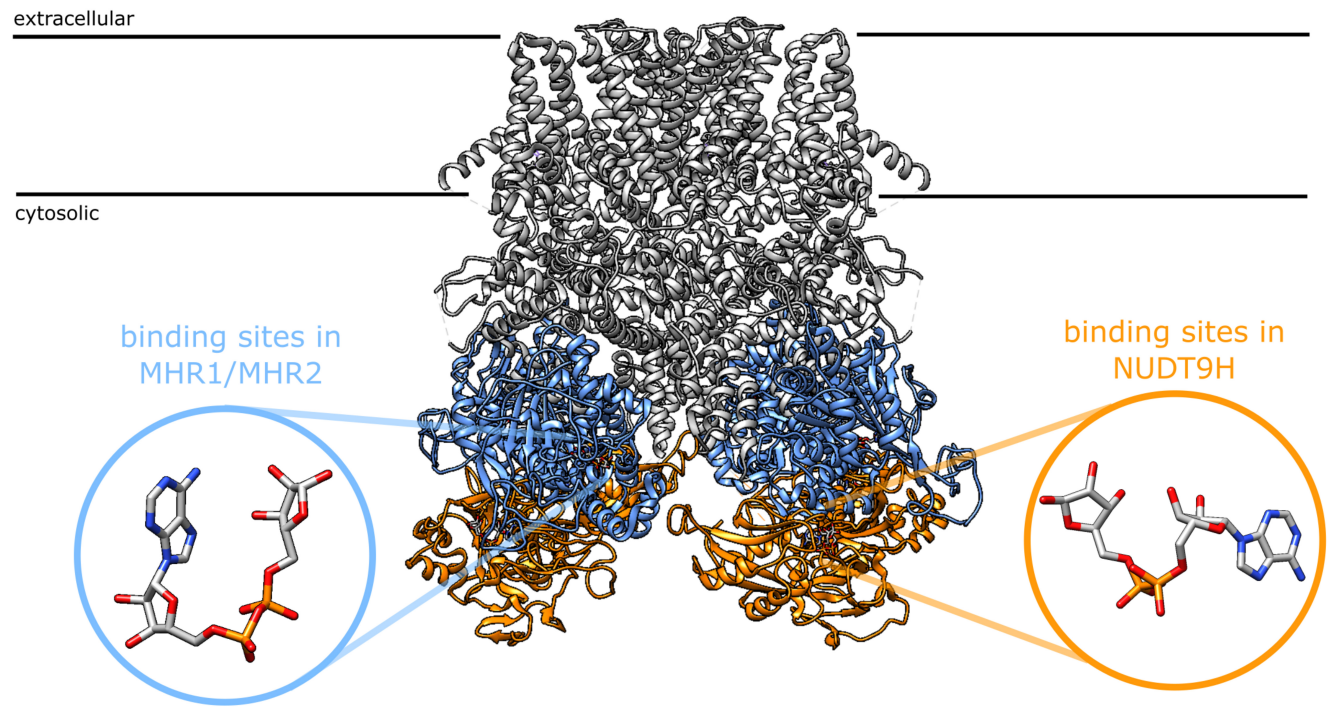

FIGURE 1 | Domain structure of TRPM2 [after (46)] and location of the two nucleotide binding sites in the cryoEM structure of human TRPM2 [pdb: 6PUS, (48)]: the recently identified N-terminal binding site between the MHR1 and MHR2 domains (blue) and the established C-terminal binding site in the NudT9H domain. The insets show the different conformation of ADPR in the binding sites as determined from the cryoEM structure, the ADPR in the N-terminal binding site assumes a horseshoe like conformation whereas the ADPR in in the NudT9H domain has a more elongated conformation. The depicted ADPR molecules have been reoriented relative to the TRPM2 structure to better illustrate the difference in conformation between the two binding sites. In a structure that has been solved in the presence of 8-Br-cADPR, 8-Br-cADPR occupies the N-terminal binding site between MHR1/MHR2 (48).

without production of ADPR (54). Increasing temperature accelerates chemical and enzymatic turnover but due to the rapid kinetics it appears unlikely that increased hydrolysis of cADPR to ADPR is responsible for the results observed by Togashi et al. In addition $\mathrm{Yu}$ et al. demonstrated that wildtype HEK293 cells do not express CD38 or CD157/Bst-1 nor do they show turnover of CADPR to ADPR over the time course of a typical patch-clamp experiment (55).

\section{CONTAMINATION OF COMMERCIAL CADPR PREPARATIONS}

A complicating factor in interpreting the results from Kolisek et al. and Togashi et al. is, that commercial preparations of cADPR from one of the major suppliers are often partially degraded and contain significant amounts of ADPR. Heiner et al. noticed high currents when infusing CADPR into human neutrophils which prompted them to check their solutions for ADPR contaminations by HPLC (56). They found that even freshly prepared solutions from several batches of commercial cADPR, contained roughly $25 \%$ ADPR. When they incubated the contaminated CADPR with nucleotide pyrophosphatase thereby fully converting ADPR to AMP and ribose 5-phosphate, the ADPR-free cADPR did no longer evoke TRPM2 currents in the granulocytes (56). When using a commercial preparation of cADPR Tóth et al. also observed activation of TRPM2 by "CADPR" in inside-out patches from Xenopus oocytes, but analysis of the composition of the "CADPR" preparation by thin layer chromatography showed that in addition to CADPR it contained roughly 20\% ADPR (57). Selective hydrolysis of ADPR by nucleotide pyrophosphatase, without degradation of cADPR (58), resulted in a complete loss of TRPM2 activation. Both groups noticed that the loss in channel activation was not due to inhibition by AMP as addition of the same amount of AMP to ADPR did not affect activation of the channel by $\operatorname{ADPR}(56,57)$. Interestingly, in contrast to previous reports that showed inhibition by AMP with an $\mathrm{IC}_{50}$ of $70 \mu \mathrm{M}$ (24) and later of $10 \mu \mathrm{M}$ (8) Tóth et al. didn't observe any inhibition of human TRPM2 expressed in Xenopus oocytes by AMP up to $200 \mu \mathrm{M}$ (57).

Like for cADPR commercial preparations also 8-Br-cADPR often contains significant amounts of 8 -Br-ADPR (>20\%). The observation that 8 -Br-ADPR is a low affinity competitive antagonist for ADPR on TRPM2 (7) [ IC $_{50} \sim 300 \mu \mathrm{M}$ (18)] further complicates interpretation of reports of selective inhibition of cADPR-mediated activation of TRPM2 (24). One conceivable explanation for these results might be that the administration of an excess of 8-Br-ADPR (from the 8-Br-cADPR) to a small amount of ADPR (as a contaminant in cADPR) is effectively preventing channel activation, while it has no effect on activation of TRPM2 by $100 \mu \mathrm{M}$ ADPR. This could also explain how 8-Br-cADPR exerts its effects on $\mathrm{H}_{2} \mathrm{O}_{2}$ mediated activation of TRPM2 (24). Interestingly it has been 
shown recently that a variant of nvTRPM2 lacking the NudT9H domain can be activated by 8 -Br-ADPR acting as a low affinity partial agonist (59), indicating that 8-Br-ADPR may bind to the $\mathrm{N}$-terminal nucleotide binding domain of TRPM2. This and the amount of 8-Br-ADPR in commercial preparations of 8-BrCADPR raises the question whether the resolution of the current cryo-EM structures is sufficient to exclude the possibility that the nucleotide observed in the $\mathrm{N}$-terminal binding site in the $\mathrm{pdb}$ structure $6 \mathrm{PUU}$ (48) is not 8 - $\mathrm{Br}$-cADPR but 8 -Br-ADPR in a horseshoe-like conformation.

Interestingly a relatively recent paper by $\mathrm{Yu}$ et al. again seems to demonstrate activation of human TRPM2 overexpressed in HEK cells by cADPR (55). The concentration-response curve for cADPR was shifted to the right with an $\mathrm{EC}_{50}$ of $250 \mu \mathrm{M}$ compared to $40 \mu \mathrm{M}$ for ADPR. In stark contrast to what has been observed by Kolisek et al. (24) the maximal currents for ADPR and CADPR were similar (55). They tried to account for the problems with ADPR contaminations described above by using CADPR they either synthesized themselves or purified from commercial cADPR and demonstrated purity by mass spectrometry. Although the previous data by Kolisek et al. indicated that cADPR binds to a different site than ADPR they assumed binding to the NUDT9H domain which they confirmed by showing the binding to the isolated NUDT9H domain using surface plasmon resonance. Using molecular dynamics simulation they identified a number of residues involved in binding to cADPR and ADPR. Mutations of some of these residues exhibited differential effects on channel activation by either ligand (55). It is really interesting to see, that even more than 10 years after Heiner et al. (56) and Tóth et al. (57) convincingly demonstrated that removal of contaminating ADPR prevents activation of TRPM2 by commercial cADPR, the idea that cADPR could affect TRPM2 still lingers on.

\section{REFERENCES}

1. Yamamoto S, Shimizu S, Kiyonaka S, Takahashi N, Wajima T, Hara Y, et al. TRPM2-mediated $\mathrm{Ca}^{2+}$ influx induces chemokine production in monocytes that aggravates inflammatory neutrophil infiltration. Nat Med. (2008) 14:73847. doi: $10.1038 / \mathrm{nm} 1758$

2. Shimizu S, Yonezawa R, Negoro T, Yamamoto S, Numata T, Ishii M, et al. Sensitization of $\mathrm{H}_{2} \mathrm{O}_{2}$-induced TRPM2 activation and subsequent interleukin8 (CXCL8) production by intracellular $\mathrm{Fe}^{2+}$ in human monocytic U937 cells. Int J Biochem Cell Biol. (2015) 68:119-27. doi: 10.1016/j.biocel.2015.09.005

3. Kashio M, Sokabe T, Shintaku K, Uematsu T, Fukuta N, Kobayashi N, et al. Redox signal-mediated sensitization of transient receptor potential melastatin 2 (TRPM2) to temperature affects macrophage functions. Proc Natl Acad Sci USA. (2012) 109:6745-50. doi: 10.1073/pnas.1114193109

4. Zou J, Ainscough JF, Yang W, Sedo A, Yu S-P, Mei Z-Z, et al. A differential role of macrophage TRPM2 channels in $\mathrm{Ca}^{2+}$ signaling and cell death in early responses to H2O2. Am J Physiol Cell Physiol. (2013) 305:C61-9. doi: 10.1152/ajpcell.00390.2012

5. Yao H, Zhang Y, Liu L, Xu Y, Liu X, Lin J, et al. Inhibition of lanthanide nanocrystal-induced inflammasome activation in macrophages by a surface coating peptide through abrogation of ROS production and TRPM2-mediated $\mathrm{Ca}(2+)$ influx. Biomaterials. (2016) 108:143-56. doi: 10.1016/j.biomaterials. 2016.08.036

6. Wehage E, Eisfeld J, Heiner I, Jüngling E, Zitt C, Lückhoff A. Activation of the cation channel long transient receptor potential channel 2 (LTRPC2) by hydrogen peroxide. A splice variant reveals a mode of activation
To avoid misleading results in the future, we consider it of utmost importance to always keep in mind both, the possibility that commercial preparations of cADPR can contain significant amounts of ADPR (even despite the advertised purity) and the limited stability of cADPR in solution, even when frozen. When working with CADPR we would therefore highly recommend to (i) purify commercial preparations before use, and (ii) to test for degradation of cADPR in solution routinely by using a suitable HPLC system.

\section{AUTHOR CONTRIBUTIONS}

All authors listed have made a substantial, direct and intellectual contribution to the work, and approved it for publication.

\section{FUNDING}

This work was supported by the Deutsche Forschungsgemeinschaft (DFG) (Project number 335447717; SFB1328, project A01 to AG, SFB1328, project A05 to RF). Research in the Guse/Fliegert labs is also supported by the Joachim-HerzFoundation, the Infectophysics consortium, project 4; and EU project INTEGRATA - DLV-813284.

\section{ACKNOWLEDGMENTS}

Visualization of the structures was done using UCSF Chimera, developed by the Resource for Biocomputing, Visualization, and Informatics at the University of California, San Francisco, with support from NIH P41-GM103311 (60).

independent of ADP-ribose. J Biol Chem. (2002) 277:23150-6. doi: 10.1074/ jbc.M112096200

7. Partida-Sanchez S, Gasser A, Fliegert R, Siebrands CC, Dammermann W, Shi $\mathrm{G}$, et al. Chemotaxis of mouse bone marrow neutrophils and dendritic cells is controlled by adp-ribose, the major product generated by the CD38 enzyme reaction. J Immunol. (2007) 179:7827-39. doi: 10.4049/jimmunol.179.11.7827

8. Lange I, Penner R, Fleig A, Beck A. Synergistic regulation of endogenous TRPM2 channels by adenine dinucleotides in primary human neutrophils. Cell Calcium. (2008) 44:604-15. doi: 10.1016/j.ceca.2008.05.001

9. Robledo-Avila FH, Ruiz-Rosado de JD, Brockman KL, Partida-Sánchez S. The TRPM2 ion channel regulates inflammatory functions of neutrophils during listeria monocytogenes infection. Front Immunol. (2020) 11:97. doi: 10.3389/ fimmu.2020.00097

10. Sumoza-Toledo A, Lange I, Cortado H, Bhagat H, Mori Y, Fleig A, et al. Dendritic cell maturation and chemotaxis is regulated by TRPM2-mediated lysosomal Ca2+ release. FASEB J. (2011) 25:3529-42. doi: 10.1096/fj.10178483

11. Melzer N, Hicking G, Göbel K, Wiendl H. TRPM2 cation channels modulate T cell effector functions and contribute to autoimmune CNS inflammation. PLoS One. (2012) 7:e47617. doi: 10.1371/journal.pone.0047617

12. Wang G, Cao L, Liu X, Sieracki NA, Di A, Wen X, et al. Oxidant sensing by TRPM2 inhibits neutrophil migration and mitigates inflammation. Dev Cell. (2016) 38:453-62. doi: 10.1016/j.devcel.2016.07.014

13. Togashi K, Hara Y, Tominaga T, Higashi T, Konishi Y, Mori Y, et al. TRPM2 activation by cyclic ADP-ribose at body temperature is involved in insulin secretion. EMBO J. (2006) 25:1804-15. doi: 10.1038/sj.emboj.7601083 
14. Starkus JG, Fleig A, Penner R. The calcium-permeable non-selective cation channel TRPM2 is modulated by cellular acidification. J Physiol. (2010) 588:1227-40. doi: 10.1113/jphysiol.2010.187476

15. McHugh D, Flemming R, Xu S-Z, Perraud A-L, Beech DJ. Critical intracellular $\mathrm{Ca}^{2+}$ dependence of transient receptor potential melastatin 2 (TRPM2) cation channel activation. J Biol Chem. (2003) 278:11002-6. doi: 10.1074/jbc. M210810200

16. Starkus J, Beck A, Fleig A, Penner R. Regulation of TRPM2 by extra- and intracellular calcium. J Gen Physiol. (2007) 130:427-40. doi: 10.1085/jgp. 200709836

17. Nagamine K, Kudoh J, Minoshima S, Kawasaki K, Asakawa S, Ito F, et al. Molecular cloning of a novel putative $\mathrm{Ca}^{2+}$ channel protein (TRPC7) highly expressed in brain. Genomics. (1998) 54:124-31. doi: 10.1006/geno.1998. 5551

18. Fliegert R, Bauche A, Wolf Pérez A-M, Watt JM, Rozewitz MD, Winzer $\mathrm{R}$, et al. 2'-Deoxyadenosine $5^{\prime}$-diphosphoribose is an endogenous TRPM2 superagonist. Nat Chem Biol. (2017) 13:1036-44. doi: 10.1038/nchembio.2415

19. Perraud AL, Fleig A, Dunn CA, Bagley LA, Launay P, Schmitz C, et al. ADPribose gating of the calcium-permeable LTRPC2 channel revealed by Nudix motif homology. Nature. (2001) 411:595-9. doi: 10.1038/35079100

20. Srouji JR, Xu A, Park A, Kirsch JF, Brenner SE. The evolution of function within the Nudix homology clan. Proteins. (2017) 85:775-811. doi: 10.1002/ prot. 25223

21. Howard M, Grimaldi JC, Bazan JF, Lund FE, Santos-Argumedo L, Parkhouse RM, et al. Formation and hydrolysis of cyclic ADP-ribose catalyzed by lymphocyte antigen CD38. Science. (1993) 262:1056-9. doi: 10.1126/science. 8235624

22. Zocchi E, Franco L, Guida L, Benatti U, Bargellesi A, Malavasi F, et al. A single protein immunologically identified as $\mathrm{CD} 38$ displays $\mathrm{NAD}^{+}$glycohydrolase, ADP-ribosyl cyclase and cyclic ADP-ribose hydrolase activities at the outer surface of human erythrocytes. Biochem Biophys Res Commun. (1993) 196:1459-65. doi: 10.1006/bbrc.1993.2416

23. Nikiforov A, Kulikova V, Ziegler M. The human NAD metabolome: functions, metabolism and compartmentalization. Crit Rev Biochem Mol Biol. (2015) 50:284-97. doi: 10.3109/10409238.2015.1028612

24. Kolisek M, Beck A, Fleig A, Penner R. Cyclic ADP-ribose and hydrogen peroxide synergize with ADP-ribose in the activation of TRPM2 channels. $\mathrm{Mol}$ Cell. (2005) 18:61-9. doi: 10.1016/j.molcel.2005.02.033

25. Lee HC. Cyclic ADP-ribose and nicotinic acid adenine dinucleotide phosphate (NAADP) as messengers for calcium mobilization. I Biol Chem. (2012) 287:31633-40. doi: 10.1074/jbc.R112.349464

26. Guse AH, da Silva CP, Berg I, Skapenko AL, Weber K, Heyer P, et al. Regulation of calcium signalling in $\mathrm{T}$ lymphocytes by the second messenger cyclic ADP-ribose. Nature. (1999) 398:70-3. doi: 10.1038/18024

27. Partida-Sánchez S, Cockayne DA, Monard S, Jacobson EL, Oppenheimer N, Garvy B, et al. Cyclic ADP-ribose production by CD38 regulates intracellular calcium release, extracellular calcium influx and chemotaxis in neutrophils and is required for bacterial clearance in vivo. Nat Med. (2001) 7:1209-16. doi: 10.1038/nm1101-1209

28. Walseth TF, Aarhus R, Kerr JA, Lee HC. Identification of cyclic ADPribose-binding proteins by photoaffinity labeling. J Biol Chem. (1993) 268:26686-91.

29. Fritz N, Macrez N, Mironneau J, Jeyakumar LH, Fleischer S, Morel JL. Ryanodine receptor subtype 2 encodes $\mathrm{Ca}^{2+}$ oscillations activated by acetylcholine via the M2 muscarinic receptor/cADP-ribose signalling pathway in duodenum myocytes. J Cell Sci. (2005) 118:2261-70. doi: 10.1242/jcs. 02344

30. Dabertrand F, Fritz N, Mironneau J, Macrez N, Morel J-L. Role of RYR3 splice variants in calcium signaling in mouse nonpregnant and pregnant myometrium. Am J Physiol Cell Physiol. (2007) 293:C848-54. doi: 10.1152/ ajpcell.00069.2007

31. Noguchi N, Takasawa S, Nata K, Tohgo A, Kato I, Ikehata F, et al. Cyclic ADP-ribose binds to FK506-binding protein 12.6 to release $\mathrm{Ca}^{2+}$ from islet microsomes. J Biol Chem. (1997) 272:3133-6. doi: 10.1074/jbc.272.6.3133

32. Zhang X, Tallini YN, Chen Z, Gan L, Wei B, Doran R, et al. Dissociation of FKBP12.6 from ryanodine receptor type 2 is regulated by cyclic ADP-ribose but not beta-adrenergic stimulation in mouse cardiomyocytes. Cardiovasc Res. (2009) 84:253-62. doi: $10.1093 /$ cvr/cvp212
33. Yamasaki-Mann M, Demuro A, Parker I. cADPR stimulates SERCA activity in Xenopus oocytes. Cell Calcium. (2009) 45:293-9. doi: 10.1016/j.ceca.2008.11. 008

34. Yamasaki-Mann M, Demuro A, Parker I. Modulation of endoplasmic reticulum $\mathrm{Ca}^{2+}$ store filling by cyclic ADP-ribose promotes inositol trisphosphate $\left(\mathrm{IP}_{3}\right)$-evoked $\mathrm{Ca}^{2+}$ signals. J Biol Chem. (2010) 285:25053-61. doi: 10.1074/jbc.M109.095257

35. Park D-R, Nam T-S, Kim Y-W, Lee S-H, Kim U-H. CD38-cADPR-SERCA signaling axis determines skeletal muscle contractile force in response to $\beta$ adrenergic stimulation. Cell Physiol Biochem. (2018) 46:2017-30. doi: 10.1159/ 000489441

36. Guse AH, Berg I, Da Silva CP, Potter BVL, Mayr GW. Ca ${ }^{2+}$ entry induced by cyclic ADP-ribose in intact T-lymphocytes. J Biol Chem. (1997) 272:8546-50. doi: $10.1074 /$ jbc.272.13.8546

37. Kiselyov K, Shin DM, Shcheynikov N, Kurosaki T, Muallem S. Regulation of $\mathrm{Ca}^{2+}$-release-activated $\mathrm{Ca}^{2+}$ current $\left(\mathrm{I}_{\text {crac }}\right)$ by ryanodine receptors in inositol 1,4,5-trisphosphate-receptor-deficient DT40 cells. Biochem J. (2001) 360:1722. doi: 10.1042/0264-6021:3600017

38. da Silva CP, Potter BVL, Mayr GW, Guse AH. Quantification of intracellular levels of cyclic ADP-ribose by high-performance liquid chromatography. J Chromatogr B Biomed Sci Appl. (1998) 707:43-50. doi: 10.1016/S03784347(97)00622-1

39. Graeff R, Lee HC. A novel cycling assay for cellular cADP-ribose with nanomolar sensitivity. Biochem J. (2002) 361:379-84. doi: 10.1042/bj3610379

40. Young GS, Kirkland JB. Modifications to increase the efficiency of the fluorometric cycling assay for cyclic ADP-ribose. Comb Chem High Throughput Screen. (2006) 9:633-7. doi: 10.2174/138620706778249749

41. Bruzzone S, Guse AH. Cycling assay for determining intracellular cyclic adpribose levels. Cold Spring Harb Protoc. (2013) 2013:564-8. doi: 10.1101/pdb. prot072991

42. Walseth TF, Lee HC. Synthesis and characterization of antagonists of cyclicADP-ribose-induced $\mathrm{Ca}^{2+}$ release. Biochim Biophys Acta. (1993) 1178:235-42.

43. Kühn FJP, Kühn C, Lückhoff A. Functional characterisation of a TRPM2 orthologue from the sea anemone Nematostella vectensis in human cells. Sci Rep. (2015) 5:8032. doi: 10.1038/srep08032

44. Kühn FJP, Watt JM, Potter BVL, Lückhoff A. Different substrate specificities of the two ADPR binding sites in TRPM2 channels of Nematostella vectensis and the role of IDPR. Sci Rep. (2019) 9:4985. doi: 10.1038/s41598-019-41531-4

45. Zhang Z, Tóth B, Szollosi A, Chen J, Csanády L. Structure of a TRPM2 channel in complex with $\mathrm{Ca}^{2+}$ explains unique gating regulation. Elife. (2018) 7:e36409. doi: 10.7554/eLife.36409

46. Wang L, Fu T-M, Zhou Y, Xia S, Greka A, Wu H. Structures and gating mechanism of human TRPM2. Science. (2018) 362:eaav4809. doi: 10.1126/ science.aav4809

47. Huang Y, Winkler PA, Sun W, Lü W, Du J. Architecture of the TRPM2 channel and its activation mechanism by ADP-ribose and calcium. Nature. (2018) 562:145-9. doi: 10.1038/s41586-018-0558-4

48. Huang Y, Roth B, Lü W, Du J. Ligand recognition and gating mechanism through three ligand-binding sites of human TRPM2 channel. Elife. (2019) 8:e50175. doi: 10.7554/eLife.50175

49. Zhang FJ, Gu QM, Sih CJ. Bioorganic chemistry of cyclic ADP-ribose (cADPR). Bioorganic Med Chem. (1999) 7:653-64. doi: 10.1016/S09680896(98)00256-9

50. Jacobson MK, Coyle DL, Vu CQ, Kim H, Jacobson EL. Preparation of cyclic ADP-ribose, 2'-phospho-cyclic ADP-ribose, and nicotinate adenine dinucleotide phosphate: possible second messengers of calcium signaling. Methods Enzymol. (1997) 280:265-275. doi: 10.1016/S0076-6879(97)80118-8

51. Lee HC, Zocchi E, Guida L, Franco L, Benatti U, De Flora A. Production and hydrolysis of cyclic ADP-ribose at the outer surface of human erythrocytes. Biochem Biophys Res Commun. (1993) 191:639-45.

52. Takasawa S, Tohgo A, Noguchi N, Koguma T, Nata K, Sugimoto T, et al. Synthesis and hydrolysis of cyclic ADP-ribose by human leukocyte antigen CD38 and inhibition of the hydrolysis by ATP. J Biol Chem. (1993) 268:26052-4.

53. Yamamoto-Katayama S, Ariyoshi M, Ishihara K, Hirano T, Jingami $H$, Morikawa K. Crystallographic studies on human BST-1/CD157 with ADPribosyl cyclase and NAD glycohydrolase activities. J Mol Biol. (2002) 316:71123. doi: $10.1006 /$ jmbi.2001.5386 
54. Canales J, Fernández A, Rodrigues JR, Ferreira R, Ribeiro JM, Cabezas A, et al. Hydrolysis of the phosphoanhydride linkage of cyclic ADP-ribose by the Mn2+-dependent ADP-ribose/CDP-alcohol pyrophosphatase. FEBS Lett. (2009) 583:1593-8. doi: 10.1016/j.febslet.2009.04.023

55. Yu P, Liu Z, Yu X, Ye P, Liu H, Xue X, et al. Direct Gating of the TRPM2 channel by CADPR via specific interactions with the ADPR binding pocket. Cell Rep. (2019) 27:3684-95.e4. doi: 10.1016/j.celrep.2019. 05067

56. Heiner I, Eisfeld J, Warnstedt M, Radukina N, Jüngling E, Lückhoff A. Endogenous ADP-ribose enables calcium-regulated cation currents through TRPM2 channels in neutrophil granulocytes. Biochem J. (2006) 398:225-32. doi: 10.1042/BJ20060183

57. Tóth B, Csanády L. Identification of direct and indirect effectors of the transient receptor potential melastatin 2 (TRPM2) cation channel. J Biol Chem. (2010) 285:30091-102. doi: 10.1074/jbc.M109.066464

58. Gu QM, Sih CJ. Cyclic ADP-ribose: synthesis and structural assignment. J Am Chem Soc. (1994) 116:7481-6. doi: 10.1021/ja00096a002
59. Tóth B, Iordanov I, Csanády L. Selective profiling of N-And C-terminal nucleotide-binding sites in a TRPM2 channel. J Gen Physiol. (2020) 152:1-13. doi: 10.1085/JGP.201912533

60. Pettersen EF, Goddard TD, Huang CC, Couch GS, Greenblatt DM, Meng EC, et al. Chimera - a visualization system for exploratory research and analysis. $J$ Comput Chem. (2004) 25:1605-12. doi: 10.1002/jcc.20084

Conflict of Interest: The authors declare that the research was conducted in the absence of any commercial or financial relationships that could be construed as a potential conflict of interest.

Copyright (c) 2020 Fliegert, Riekehr and Guse. This is an open-access article distributed under the terms of the Creative Commons Attribution License (CC BY). The use, distribution or reproduction in other forums is permitted, provided the original author(s) and the copyright owner(s) are credited and that the original publication in this journal is cited, in accordance with accepted academic practice. No use, distribution or reproduction is permitted which does not comply with these terms. 